\title{
Fabrication of Nanopores Using Electron Beam
}

\author{
Tao Xu, Xiao Xie, Litao Sun* \\ SEU-FEI Nano-Pico Center, Key Laboratory of MEMS of Ministry of Education, Southeast University, CHINA \\ *Corresponding Author: Litao Sun, slt@seu.edu.cn
}

\begin{abstract}
Sub-5 $\mathrm{nm}$ nanopores are widely used in singlemolecule detections for biological and chemical applications. However, the traditional fabrication methods are difficult to reduce pore size to sub-5 $\mathrm{nm}$. Focused electron beam irradiation inside a transmission electron microscope (TEM) is verified experimentally to be an effectively straightforward way to fabricate such small pores. The fabrication process can be explained by a simple physical collision model. We also fabricate graphene nanopores under various temperatures. The results verify that drilling at high temperature and direct thermal heating treatment can be beneficial to fabricate carbon nanostructure with high crystallization, which will promote the study of biological measurement. High temperature could also modulate the duration of fabrication, which can enhance the size control during nanopore fabrication.
\end{abstract}

Keywords-nanopore; TEM; fabrication; electron beam irradiation

\section{INTRODUCTION}

Sub-5 nm nanopores embedded in thin membrane have attracted worldwide attention due to its potential to singlemolecular detection and identification. It has been reported that a nanopore of $1-5 \mathrm{~nm}$ is suitable for characterizing most of biomolecules[1-4]. For example, solid-state nanopores in some thin membranes (silicon oxide[5], silicon nitride[6-7], aluminum oxide[8-9] and graphene[10-12]) have been used to detect single-strand DNA. How to fabricate sub-5 nm nanopores is a basic and key problem. Although many methods (magnetron sputtering, chemical etching, ion beam induced sputtering, etc) were developed to fabricate nanopores, it is difficult to reduce the nanopore size to sub-5 nm. Nevertheless, electron beam irradiation inside a transmission electron beam (TEM) has become the most effective way to obtain such small pores[13-19]. Previous experiments[13-15] indicate that electron beam size is one of the limiting factor to ultimate pore size, and the beam size could be focused to several nanometer even sub-nanometer inside a TEM. On the other hand, nanopore size could be modulated by electron beam irradiation at optimized electron intensity to reduce the surface free energy and to reconstruct more stable nanostructure, which is possible to reduce the pore size to 5 nm, even closure[16-19]. However, electron beam irradiation would inevitably damage the crystalline structure of the membrane, causing amorphization of the substrate. More severely, the damaged structures may increase the noise of the nanopore-based devices which limit their applications on biological molecules detection and analysis. Direct thermal heating without electron irradiation could assist to modulate the morphology of nanopores[20-21]. Moreover, irradiation damage can be avoided or minimized by the heat effect because heat treatments may promote the self-repairing of sample, especially on the carbon nanomaterials[22-23].

In this paper, we tried to drill nanopores in various membranes using focused electron beam inside a high resolution TEM. A simple model indicates that nanopores could be fabricated in many materials, which is in agreement with the experimental results. We also fabricated graphene nanopores at various temperatures. The results also verify that drilling at high temperature and direct thermal heating treatment can be beneficial to fabricate carbon nanostructure with high crystallization, which may promote the study of biological applications.

\section{RESULTS AND DISCUSSION}

All nanopores were fabricated in a high resolution TEM (FEI Titan 80-300) with a field emission gun operating at 300 $\mathrm{kV}$ accelerating voltage. To in-situ heat the sample, a heating sample holder (Gatan $^{\text {TM }}$ 628) was used. To remove the contamination induced by adsorbed hydrocarbon molecules, the specimen was kept at $300^{\circ} \mathrm{C}$ for $30 \mathrm{~min}$ before fabrication[24]. When the electron beam was focused into several nanometers with a Gaussian dispersion, the average current density is estimated to be $10^{3} \sim 10^{5} \mathrm{~A} / \mathrm{cm}^{2}$.

In order to show that the electron beam irradiation fabrication method can be applied to various materials, we tried to drill sub-5 nm nanopores at room temperature (RT) in graphene, $\mathrm{MoS}_{2}, \mathrm{Si}_{3} \mathrm{~N}_{4}, \mathrm{ZnO}$, as shown in Fig. 1. The dominant effect during the drilling process is knock-on (displacement) damage although effects of heating, electrostatic charging and ionization damage are also important. Knock-on displacement of atoms arises from the high-angle elastic scattering. Generally, the incident electron could transfer an amount of energy to atoms in the specimen by elastic collision. Electron beam induced sputtering occurs when the maximum transferred energy is larger than the surface displacement threshold energy. In fact, the threshold incident energy for electron sputtering is below $300 \mathrm{keV}$ for most of existing elements[24]. Hence, electron beam induced atomic displacement or sputtering under $300 \mathrm{keV}$ electron beam can become an important way to fabricate sub-5 nm nanopores in many materials.

Although nanopores in $\mathrm{Si}_{3} \mathrm{~N}_{4}, \mathrm{SiO}_{2}$ and $\mathrm{Al}_{2} \mathrm{O}_{3}$ exhibit high stability, these membranes are too thick to obtain precise information of biomolecules (e. g., sequence of base pairs of a DNA). Due to exceptional mechanical properties and subnanometer thickness $(0.34 \mathrm{~nm})$ per layer, graphene nanopores open up the possibility to determine sequential details of DNA. Hence, we focus on the fabrication of graphene nanopores. We 

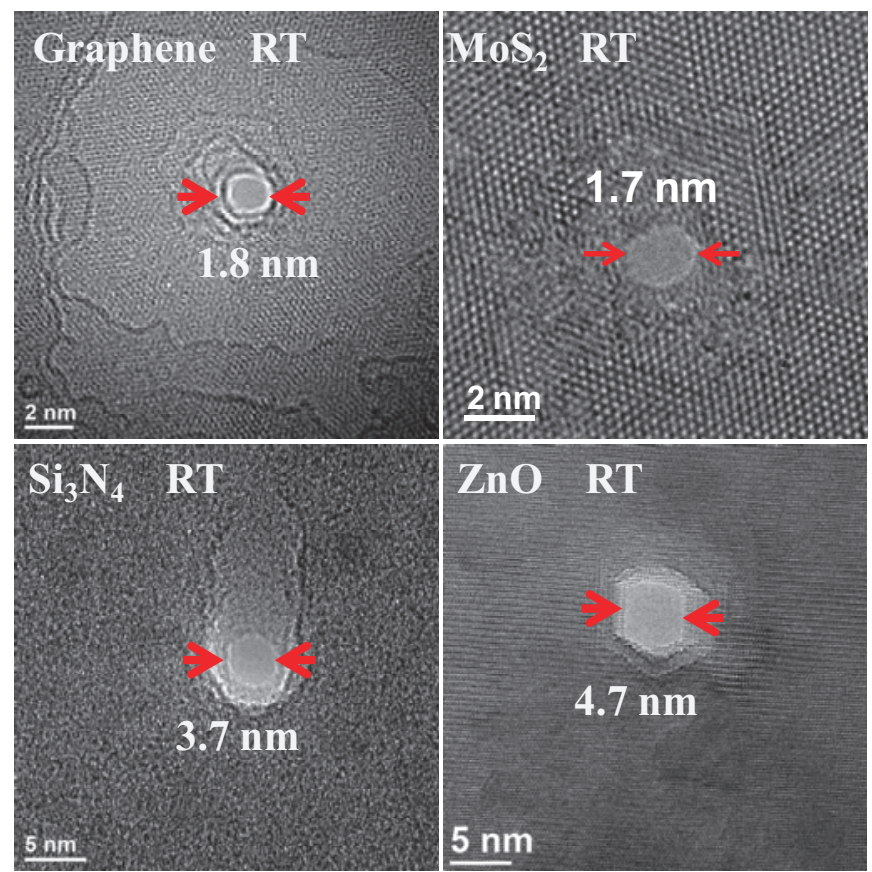

Fig. 1. Sub-5 nm nanopores on various materials.

drilled nanopores on graphene not only at room temperature but also at the temperature higher than $300^{\circ} \mathrm{C}$, because high temperature could be helpful to avoid the damages mentioned above[22-23].

To understand the fabrication process, we record the relationship between nanopore diameter and irradiation time using same beam condition at $350{ }^{\circ} \mathrm{C}$. Fig. 2 shows the size of graphene nanopores as a function of irradiation time and images of nanopores at various irradiation durations. As shown in the figure, longer irradiation time formed larger nanopores, but the diameter increased nonlinearly with the time. The procedure consists of three stages: (I) nanopore formation, (II) quick expansion, and (III) slow expansion, which is in agreement with previous literature[18]. The phenomenon is attributed to the density distribution of electron beam, which fits Gaussian distribution with a maximum density of $\sim 10^{4} \mathrm{~A} / \mathrm{cm}^{2}$ at the center of electron beam. The current density decays rapidly in the position away from the beam center. The time $T$ needed to form a nanopore is reciprocal to the current density $J$ at the fringe of the pore on the common assumption that the amount of irradiation damage is proportional to the electron dose. In other words, $1 / T$ also subjects to Gaussian distribution in the irradiation region, which shows good agreement with the experimental result. The result can also verify the assumption. As shown in Fig. 2, 21 seconds is needed to form a nanopore on graphene membrane with a thickness of $\sim 3.4 \mathrm{~nm}$. Then the nanopore enlarges quickly in another 9 seconds after pore formed because the density changes quickly along radial direction of the beam. The pore size is sensitive to irradiation time in this stage. Finally, the pore diameter increases almost linearly with the irradiation time after $30 \mathrm{~s}$ as the current density decreases slowly. In this stage, the pore size is mainly limited by the size of the electron beam.
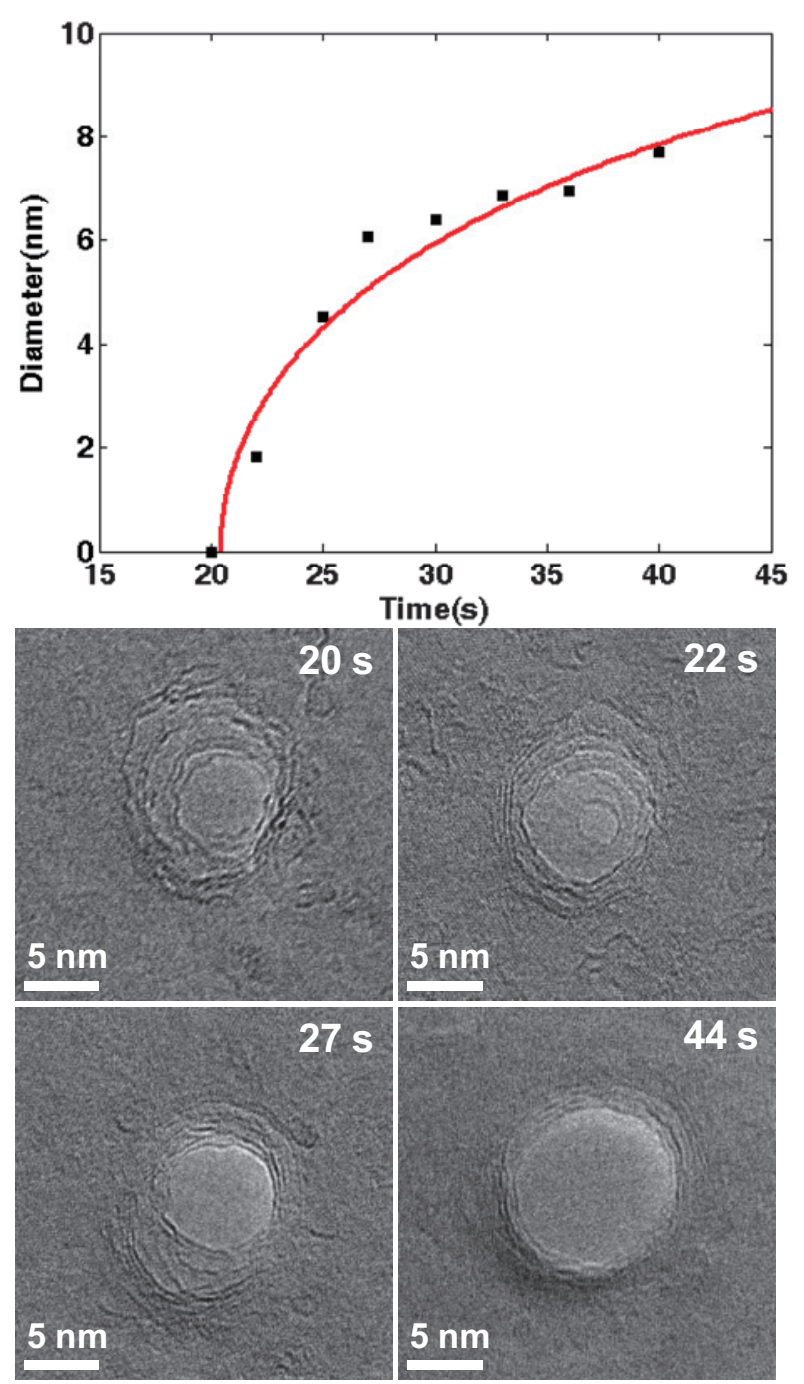

Fig. 2. Graphene nanopore diameter as a function of time and images at various irradiation time. The maximum current density of the focused electron beam is $\sim 10^{4} \mathrm{~A} / \mathrm{cm}^{2}$.

To understand the role of thermal heating during the fabrication process, we compare the nanopores drilled at various temperatures. The results indicate that the temperature has a significant effect on the drilling and radiation process under $300 \mathrm{keV}$ electron beam irradiation, as shown in Fig. 3. It is hard to obtain more information from the pore drilled at RT due to severe amorphization, which hinders high resolution imaging. But at the temperature of $300{ }^{\circ} \mathrm{C}$, the pore has a concentric ringlike structure extending several nanometers away from the edge, but the ringlike structure is not very obvious because locally amorphous structure is still presented. At the temperature of $300{ }^{\circ} \mathrm{C}$, terrace-like structures with obvious step occur around the as-fabricated nanopore. Results prove that the amorphization is effectively limited by raising the temperature of drilling process, indicating that manipulation at high temperature can fabricate highly crystallized graphene nanostructure, which is very helpful to the study of biological measurement. 

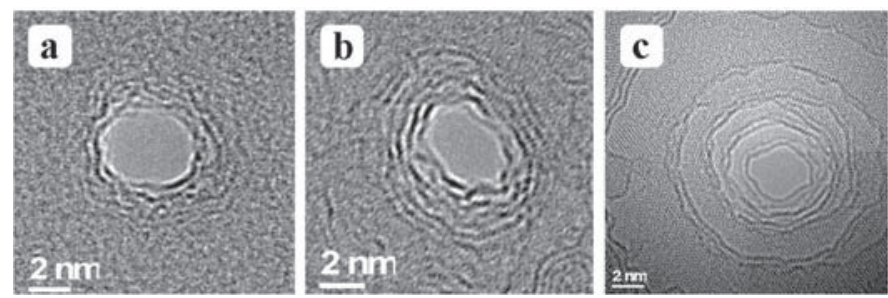

Fig. 3. Influence of temperature on the drilling of few-layers of graphene by high-energy electron beam (a) at RT, (b) at $300^{\circ} \mathrm{C}$, (c) at $450^{\circ} \mathrm{C}$. We drill pores with high current density more than $10^{4} \mathrm{~A} / \mathrm{cm}^{2}$, then radiate for several seconds with low density less than $10^{3} \mathrm{~A} / \mathrm{cm}^{2}$ to form a clear edge structure. At $\mathrm{RT}$, it is hard to identify the thickness of the graphene membrane because the area surrounding the nanopore still remains amorphous. At $300^{\circ} \mathrm{C}$, the pore has a concentric ring-like structure extending several nanometers away from the edge, but the ring-like structure is not clear enough because the local

amorphous is still presented. The edge region is clear enough at $450^{\circ} \mathrm{C}$.

Moreover, a high temperature not only promotes the crystallization of the structures around the pores but also influences the drilling rate. As shown in Fig. 4, it is obvious that the drilling rate decreased when raising the temperature, which is attributed to the self-repairing of carbon materials at high temperature. We can also find that nanopore diameters increase with increasing irradiation time at a given temperature. Due to the diminishing current density in the tails of electron beam, pore diameter can be plotted as a function of time, as in Fig. 4, which is in agreement with the function in Fig. 2. Finally, the pore size would keep constant, and the ultimate size is approximately equal to the electron beam size.

In fact, high temperature is not only important during the drilling process, but it also promotes the crystallization of the structures around the as-fabricated nanopores. As shown in Fig. 5, contrast to the behavior without thermal heating, ringlike structure occurs, extending several nanometers away from the edge of nanopore after thermal treatment. Without doubt, electron beam irradiation would generate many uncombined carbon atoms on the surface of the membrane.

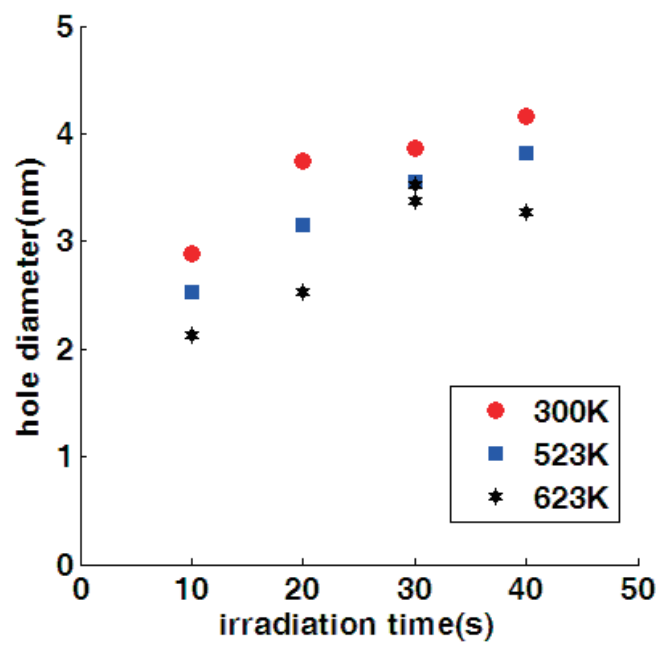

Fig. 4. Pore diameter as a function of time at different temperature, for irradiation of a graphene membrane with ahout $7 \mathrm{~nm}$ in thickness. The average current density is about $3 \times 10^{3} \mathrm{~A} / \mathrm{cm} 2$.
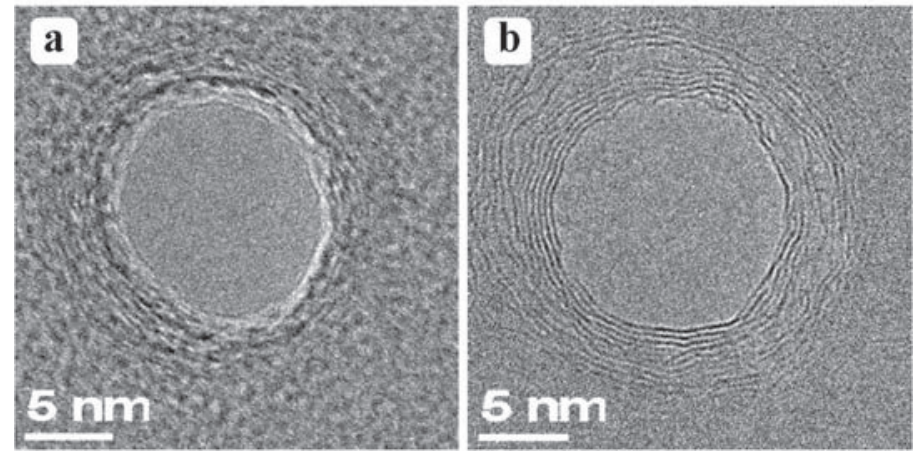

Fig.5. Influence of temperature on the holes. (a) Hole made on the RT. (b) The same area after being heated to $400^{\circ} \mathrm{C}$

These ad-atoms will migrate and form stable structures under thermal excitation.

\section{CONCLUSION}

In conclusion, sub-5 $\mathrm{nm}$ nanopores on various substrates could be fabricated using focused electron beam inside a TEM. A simple model indicates that nanopores could be fabricated in many materials, which is agreement with the experiments. We also fabricate graphene nanopores at various temperatures. The results also verify that drilling at high temperature and direct thermal heating treatment can be beneficial to fabricate carbon nanostructure with high crystallization, which may promote the study of biological measurement. High temperature could also modulate the duration of the fabrication, which can promote the size control during nanopore fabrication.

\section{ACKNOWLEDGMENT}

This work was supported by the National Basic Research Program of China (Grant Nos. 2011CB707601 and 2009CB623702), the National Natural Science Foundation of China (Nos. 61274114 and 51071044), and Chinese Postdoctoral Science Foundation (No. 2011M500081).

\section{REFERENCES}

[1] C. Dekker, "Solid-state nanopores," Nat. Nanotechnol., vol. 2, pp. 209215, April 2007

[2] S. Howorka and Z. Siwy, "Nanopore analytics: sensing of single molecules," Chem. Soc. Rev., vol. 38, pp. 2360-2384, 2009

[3] F. Olasagasti, K. R. Lieberman, S. Benner, G. M. Cherf, J. M. Dahl, D W. Deamer et al., "Replication of individual DNA molecules under electronic control using a protein nanopore," Nat. Nanotechnol., vol. 5, pp. 798-806, September 2010

[4] B. M. Venkatesan, and R. Bashir, "Nanopore sensors for nucleic acid analysis," Nat. Nanotechnol., vol. 6, pp. 615-624, September 2011

[5] H. Chang, F. Kosari, G. Andreadakis, M. A. Alam, G. Vasmatzis and R. Bashir, "DNA-Mediated Fluctuations in Ionic Current through Silicon Oxide Nanopore Channels", Nano Lett., vol. 4, pp. 1551-1556, August 2004

[6] J. Li, M. Gershow, D. Stein, E. Brandin and J. A. Golovchenko, "DNA molecules and configurations in a solid-state nanopore microscope," Nat. Mater., vol. 2, pp. 611-615, September 2003 
[7] M. Wanunu, T. Dadosh, V. Ray, J. Jin, L. McReynolds and M. Drndic, "Rapid electronic detection of probe-specific microRNAs using thin nanopore sensors," Nat. Nanotechnol., vol. 5, pp. 807-814, November 2010

[8] B. M. Venkatesan, B. Dorvel, S. Yemenicioglu, N. Watkins, I. Petrov, and R. Bashir, "Highly Sensitive, Mechanically Stable Nanopore Sensors for DNA Analysis," Adv. Mater., vol. 21, pp. 2771-2776, 2009.

[9] B. M. Venkatesan, A. B. Shah, J.-M. Zuo and R. Bashir, "DNA Sensing Using Nanocrystalline Surface-Enhanced $\mathrm{Al}_{2} \mathrm{O}_{3}$ Nanopore Sensors," Adv. Func. Mater., vol. 20, pp. 1266-1275, 2010

[10] S. Garaj, W. Hubbard, A. Reina, J. Kong, D. Branton and J. A. Golovchenko, "Graphene as a subnanometre trans-electrode membrane," Nature, vol. 467, pp. 190-193, September 2010

[11] C. A. Merchant, K. Healy, M. Wanunu, V. Ray, N. Peterman, J. Bartel, et al., "DNA Translocation through Graphene Nanopores," Nano Lett., vol. 10, pp. 2915-2921, 2010

[12] G. F. Schneider, S. W. Kowalczyk, V. E. Calado, G. Pandraud, H. W. Zandbergen, L. M. K. Vandersypen er al., "DNA Translocation through Graphene Nanopores," Nano Lett., vol. 10, pp. 3163-3167, 2010

[13] J. M. Zhang, L. P. You, H. Q. Ye and D. P. Yu, "Fabrication of ultrafine nanostructures with single-nanometre precision in a high-resolution transmission electron microscope," Nanotechnology, vol. 18, pp. 155303 April 2007

[14] T. Aref, M. Remeika and A. Bezryadina, "High-resolution nanofabrication using a highly focused electron beam," J. Appl. Phys., vol. 104, pp. 024312, July 2008

[15] M. D. Fischbein and M. Drndic, "Electron beam nanosculpting of suspended graphene sheets," Appl. Phys. Lett., vol. 93, pp. 113107, September 2008

[16] A. J. Storm, J. H. Chen, X. S. Ling, H. W. Zandbergen and C. Dekker, "Fabrication of solid-state nanopores with single-nanometre precision,", Nat. Mater., vol. 2, pp. 537-540, August 2003

[17] L. Chih Jen, A. Thomas and B. Alexey, "Fabrication of symmetric sub-5 $\mathrm{nm}$ nanopores using focused ion and electron beams," Nanotechnology, vol. 17, pp. 3264-3267, June 2006

[18] M. J. Kim, M. Wanunu, D. C. Bell and A. Meller, "Rapid Fabrication of Uniformly Sized Nanopores and Nanopore Arrays for Parallel DNA Analysis," Adv. Mater., vol. 18, pp. 3149-3153, 2006

[19] K. Min Jun, M. Ben, M. Kazuyoshi and M. Amit, "Characteristics of solid-state nanometre pores fabricated using a transmission electron microscope," Nanotechnology, vol. 18, pp. 205302, April 2007

[20] T. Xu, K. B. Yin, X. Xie, L. B. He, B. J. Wang, and L. T. Sun, "Sizedependent evolution of graphene nanopores under thermal excitation," Small, vol. 8, pp. 3422-3426, November 2012

[21] W. Asghar, A. Ilyas, J. Billo and S. Iqbal, "Shrinking of Solid-state Nanopores by Direct Thermal Heating," Nanoscale Res. Lett., vol. 6, pp. 372,2011

[22] F. Banhart, "Irradiation effects in carbon nanostructures," Rep. Prog. Phys., vol. 62, pp. 1181-1221, Auguest 1999

[23] A. V. Krasheninnikov and F. Banhart, "Engineering of nanostructured carbon materials with electron or ion beams," Nat. Mater., vol. 6, pp. 723-733, October 2007

[24] R. F. Egerton, P. Li and M. Malac, "Radiation damage in the TEM and SEM," Micron, vol. 35, pp. 399-409, 2004 